\title{
The dilemma and promise of cancer chemoprevention
}

\author{
Scott M Lippman
}

Cancer is a dreadful disease, yet drugs that reduce cancer risk are not widely accepted. Consider tamoxifen and finasteride, which significantly reduced breast cancer risk (by $49 \%$ ) and prostate cancer risk (by 25\%). ${ }^{1,2}$ Healthy, higher-risk women or men, however, seldom consider these agents, because serious side effects associated with either one ${ }^{1,2}$ have thoroughly overshadowed the benefits. Why? The multifaceted answer to this important question points to both the dilemma and the promise of cancer chemoprevention.

Cardiovascular disease (CVD) chemoprevention offers useful insights into the dilemmas of cancer chemoprevention. Statin prevention of CVD can be measured via the risk:effects marker cholesterol. There is no accepted measurable risk: effects marker for personalizing cancer chemoprevention. Conversely, low-dose aspirin is widely accepted by relatively healthy people for preventing CVD, despite lacking any measurable risk:effects marker. The reason people generally accept low-dose aspirin (which reduces CVD by only 15\%) but not tamoxifen (which reduces breast cancer by $49 \%$ ) lies in a careful consideration of disease and drug risks. 1,3 The Framingham risk model clearly indicates that low-dose aspirin is beneficial in relatively healthy people with $a \geq 1.5 \%$ annual risk of CVD. ${ }^{4}$ The side effects of low-dose aspirin are relatively few and easy to understand, consisting mainly of an increased risk of bleeding. The side effects of tamoxifen, however, are relatively many and complex, and Gail risk assessment modeling and the risk:benefit profile of tamoxifen are complicated. ${ }^{5}$ This complexity is illustrated by the Gail calculation suggesting that tamoxifen is beneficial for white women aged $50-59$ years with a uterus if they have $a \geq 6 \% 5$-year breast cancer risk. The model excludes substantial side effects, including hot flashes, vaginal discharge, and adverse sexual functioning symptoms. There is no specific Gail risk level at which tamoxifen is clearly recommended for this or any other subgroup of relatively healthy women with an

\section{The ability to \\ personalize \\ chemo- \\ prevention will \\ accelerate its \\ acceptance \\ among an \\ ever-widening \\ spectrum of \\ at-risk people}

SM Lippman is an Advisory Board member of Nature Clinical Practice Oncology.

\section{Competing interests}

The author declared he has no competing interests.

www.nature.com/clinicalpractice doi:10.1038/ncponc0609 increased risk of breast cancer (notwithstanding that the FDA approved tamoxifen for women at $a \geq 1.67 \%$ 5-year Gail risk).

The Study of Tamoxifen and Raloxifene (STAR) showed that raloxifene was as good as tamoxifen at reducing invasive breast cancer risk, and had fewer side effects. ${ }^{6}$ Although raloxifene is more toxic than low-dose aspirin, it may be sufficiently beneficial and nonharmful to become accepted by generally healthy women at risk of breast cancer. Factors that will influence the acceptability of this agent include its anti-osteoporotic effects, perceived versus actual risk, personal choice issues, liability, mortality risk, and the perception (misguided, in my opinion) that cancer chemoprevention should be a magic bullet-100\% preventive, permanent and causing no serious side effects.

Cancer chemoprevention is accepted by people at a sufficiently high perceived and actual risk level. For example, women who have had a lumpectomy and radiation for ductal carcinoma in situ often take tamoxifen, despite its side effects, to reduce a 5-year breast cancer risk that is substantially higher than the average baseline risk noted in the trial that tested the drug (Breast Cancer Prevention Trial). ${ }^{1}$

Chemoprevention with raloxifene is at the threshold of acceptance by relatively healthy women at an elevated risk of breast cancer. The ability to personalize chemoprevention will accelerate its acceptance among an everwidening spectrum of at-risk people. Genomic, proteomic and novel imaging approaches (for assessing cancer risk and preventive drug effects) and pharmaco-ecogenetic modeling based on drug interactions with environmental, metabolic and genetic factors (for predicting beneficial and adverse drug effects) are bringing personalized cancer chemoprevention closer to the practice of medicine.

Supplementary information in the form of a reference list is available on the Nature Clinical Practice Oncology website. 